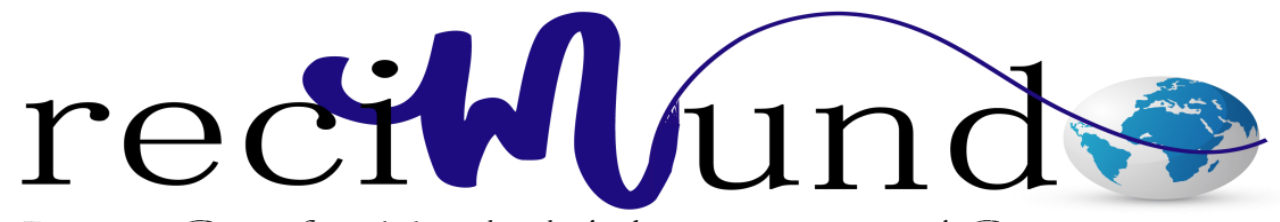

Revista Científica Mundo de la Investigación y el Conocimiento

Md José Eduardo Cedeño Gilces ${ }^{a}$; Dr. Cesar Aurelio Chalen Lainez ${ }^{\text {b; }}$ Md. Ana María Garavito Martínez ${ }^{\text {c }}$, Md. Paola Janneth Bravo Rey ${ }^{\text {d }}$

Tratamiento de la Gonartrosis mediante la aplicación de Plasma Rico en Plaquetas

Revista Científica Mundo de la Investigación y el Conocimiento. Vol. 2 núm.1, abril, ISSN: 2588-073X, 2018, pp. 1020-1032

DOI: 10.26820/recimundo/2.(2).2018.1020-1032

Editorial Saberes del Conocimiento

Recibido: 05/12/2017

Aceptado: $15 / 02 / 2018$
a. Médico de la Universidad de Guayaquil; educedenomd@ hotmail.com
b. Universidad de Guayaquil; cesar.chalenl@ug.edu.ec
c. Médico General; anamariagaravitomartinez@gmail.com
d. Médico General; p.j.bravo@hotmail.com 


\section{Tratamiento de la Gonartrosis mediante la aplicación de Plasma Rico en \\ Plaquetas}

Vol. 2, núm. 2., (2018)

Md José Eduardo Cedeño Gilces; Dr. Cesar Aurelio Chalen Lainez; Md. Ana María Garavito Martínez; Md. Paola Janneth Bravo Rey

\section{RESUMEN}

La gonartrosis u osteoartrosis de rodilla es una afección articular crónica, degenerativa, progresiva, más frecuente en mujeres, en la edad adulta, afecta la calidad de vida, por el dolor y por la limitación funcional. Aumenta los costos en los servicios de salud en el mundo. Su tratamiento abarca desde las modificaciones del estilo de vida, fármacos y cirugía, esta se considera como última instancia de tratamiento. En fármacos se utilizan analgésicos, AINES, COX 2, opiáceos vía oral o en parches. Los esteroides, Ácido Hialurónico y Plasma Rico en Plaquetas (PRP) se usan intraarticular solos o combinado. En nuestro caso de estudio, el PRP en distintos trabajos prospectivos y revisiones ha demostrado ser útil tanto en monoterapia como combinado con los otros fármacos, mejorando los síntomas y el daño articular. Por tanto se concluye que el uso de PRP es un tratamiento efectivo en la gonartrosis.

Palabras clave: Artrosis, gonartrosis, fármacos, plasma, dolor, terapia, intraarticular. 


\title{
Tratamiento de la Gonartrosis mediante la aplicación de Plasma Rico en Plaquetas
}

Vol. 2, núm. 2., (2018)

Md José Eduardo Cedeño Gilces; Dr. Cesar Aurelio Chalen Lainez; Md. Ana María Garavito Martínez; Md. Paola Janneth Bravo Rey

\begin{abstract}
Gonarthrosis or osteoarthrosis of the knee is a chronic, degenerative, progressive joint affection, more frequent in women, in adulthood; it affects quality of life, pain and functional limitation. Increase costs in health services in the world. Its treatment ranges from modifications of lifestyle, drugs and surgery; this is considered as the last instance of treatment. In drugs, analgesics, NSAIDs, COX 2, opioids are used orally or in patches. Steroids, Hyaluronic Acid and PRP are used intraarticular alone or in combination. In our case study, the PRP in different prospective studies and reviews has proven to be useful both in monotherapy and combined with other drugs, improving symptoms and joint damage. Therefore it is concluded that the use of PRP is an effective treatment in gonarthrosis.
\end{abstract}

Key words: Osteoarthritis, gonarthrosis, drugs, plasma, pain, therapy, intraarticular 


\section{Tratamiento de la Gonartrosis mediante la aplicación de Plasma Rico en \\ Plaquetas}

Vol. 2, núm. 2., (2018)

Md José Eduardo Cedeño Gilces; Dr. Cesar Aurelio Chalen Lainez; Md. Ana María Garavito Martínez; Md. Paola Janneth Bravo Rey

\section{Introducción.}

Una articulación saludable depende del balance entre una adecuada homeostasis entre el daño, reparación y remodelación; determinado por la interacción entre los distintos tipos de tejidos (cartílago, meniscos, hueso, líquido sinovial, ligamentos, tendones), cuando se altera esa homeostasis ocurre un daño que progresivamente lleva a la osteoartrosis. (1).

La osteoartrosis es una enfermedad que aumenta su prevalencia al aumentar la expectativa de vida. Hay estimaciones que $1 / 3$ de personas mayores de 35 años presentan algún signo de artrosis. En la población mayor de 65 años, uno de cada dos presenta alguna sintomatología.

En países como EEUU, Canadá, Reino Unido su costo está estimado en $2 \%$ de PIB, ocupando el segundo lugar tras enfermedades cardiovasculares con un costo similar al cáncer y mayor al de las enfermedades respiratorias. Las articulaciones más frecuentemente afectadas son las rodillas y cadera, con el consiguiente impacto en la calidad de vida. (2) (3)

Hay una amplia variedad de tratamientos para la osteoartrosis, desde distintos tratamientos no farmacológicos hasta suplementos nutricionales, terapia farmacológica, procedimientos mínimamente invasivos (inyecciones de diversas sustancias) que restauran la homeostasis mejorando la clínica y modificando el efecto de la enfermedad. Al fallar esos tratamientos es cuando se llega a los reemplazos articulares. (1)

El presente trabajo se dedica a investigar sobre la gonartrosis y la utilidad del PRP en el manejo de esa enfermedad. 


\section{Tratamiento de la Gonartrosis mediante la aplicación de Plasma Rico en Plaquetas}

Vol. 2, núm. 2., (2018)

Md José Eduardo Cedeño Gilces; Dr. Cesar Aurelio Chalen Lainez; Md. Ana María Garavito Martínez; Md. Paola Janneth Bravo Rey

\section{Metodología}

Se realizó una revisión bibliográfica de artículos de revistas, libros y/o recomendaciones de expertos en el uso de PRP versus otras sustancias para el tratamiento de la Gonartrosis.

Se procedió al análisis del material en búsqueda de información actualizada con el fin de proveer hallazgos recientes y técnicas de tratamiento para esta enfermedad.

El análisis se llevó a cabo desde la descripción de la enfermedad hasta las innovaciones terapéuticas incluidas la PRP como nueva estrategia aplicable en los pacientes.

\section{Resultados}

Gonartrosis: La gonartrosis u osteoartrosis de rodilla es una afección articular crónica, degenerativa, progresiva, que resulta de eventos mecánicos y biológicos que desestabilizan el acoplamiento normal de la articulación, es la más común en la edad adulta. Es la causa más frecuente de dolor en las rodillas o gonalgia a partir de los 40 a 50 años, afecta mayormente a mujeres $(2: 1)$ y luego de la menopausia la relación con el hombre es 1:1, suele iniciar unilateral y posteriormente se hace bilateral; es la cuarta causa de morbilidad en mujeres mayores de 60 años y la octava en los hombres. En el $75 \%$ de los pacientes el compartimiento afectado es el femorotibial interno (la rodilla está divida en tres compartimientos: compartimiento medial o interno (platillo tibial y cóndilo femoral), compartimiento lateral o externo (platillo tibial y cóndilo femoral) y la articulación patelo-femoral. Además, podemos dividir las causas en primarias y secundarias; la primaria es una aceleración del envejecimiento del cartílago y en la secundaria 


\section{Tratamiento de la Gonartrosis mediante la aplicación de Plasma Rico en \\ Plaquetas}

Vol. 2, núm. 2., (2018)

Md José Eduardo Cedeño Gilces; Dr. Cesar Aurelio Chalen Lainez; Md. Ana María Garavito Martínez; Md. Paola Janneth Bravo Rey

puede ser por diabetes, infecciones, traumática o las deformidades de la articulación que pueden ser congénitas.

Historia clínica: se interroga las características del dolor según la zona afectada, que movimiento o acción desencadena el dolor, antecedentes de lesiones traumáticas, obesidad, si es deportista o atleta. Con respecto al dolor se caracteriza por disminuir con el reposo y después del reposos puede haber rigidez articular de corta duración (15 a 30 minutos) si hay humedad o frio la rigidez es mayor y dura más tiempo.

Examen físico: se evalúa alineación de miembros inferiores, los signos asociados: derrame articular, seudobloqueos dolorosos, etc. A la historia clínica y examen físico se asocia la evaluación radiológica. La imagen solo es útil para confirmar la sospecha clínica; radiológicamente se puede observar adelgazamiento de la interlínea articular, presencia de osteofitos, esclerosis y quistes subcondrales. (4) (5) (6) (7) (8)

Para estratificar los cambios degenerativos desde el punto de vista radiológico se usan la escala de Kellgren que valora la presencia de osteofitos, tamaño y estrechamiento de la line articular y la escala Ahlback que evalúa el estrechamiento del espacio tibiofemoral. (9)

El Tratamiento: se fundamenta en los síntomas, etapa de la enfermedad, edad del paciente, ocupación, actividades diarias, sin olvidar que el tratamiento no es curativo. El objetivo es aliviar el dolor, disminuir la limitación funcional y retrasar la destrucción articular.

Tratamiento no farmacológico: el paciente debe entender que el reposo es importante y aprender a vivir con las limitaciones propias a su daño articular. Si hay sobrepeso u obesidad, el 


\section{Tratamiento de la Gonartrosis mediante la aplicación de Plasma Rico en Plaquetas}

Vol. 2, núm. 2., (2018)

Md José Eduardo Cedeño Gilces; Dr. Cesar Aurelio Chalen Lainez; Md. Ana María Garavito Martínez; Md. Paola Janneth Bravo Rey

paciente debe bajar de peso. Evitar: estar de pie por tiempos prolongados, largas caminatas, piernas cruzadas, cargar grandes pesos. Fortalecer los músculos que intervienen en esta articulación, en especial el cuádriceps. La fisioterapia antiinflamatoria puede utilizarse como coadyuvante en los períodos más dolorosos. (5)

Tratamiento farmacológico: analgésicos, AINES y COX-2 en los periodos más álgidos. Fármacos condroprotectores por vía sistémica tienen una eficacia dudosa, si bien su inocuidad hace que, en algunos pacientes, sea importante su prescripción como soporte psicológico.

\section{Plasma Rico en Plaquetas (PRP)}

Las plaquetas son células sin núcleo, el producto final de los megacariocitos, se forman en la medula ósea. Ayudan a la regeneración tisular, ayudan a la angiogenesis, procesos inflamatorios y respuesta inmune.

El PRP, es autólogo, con valores de plaquetas superior al promedio que son 200.000, es una fracción del plasma que fue centrifugado y contiene hasta 5 veces más plaquetas. El proceso de toma y manipulación de la muestra para obtener el PRP es un proceso con técnica estéril, aséptica, ya que el PRP se introduce en el organismo humano, se considera que entre la extracción de la sangre y la administración del PRP no deben pasar más de 45 minutos. (10) (11)

Usos o beneficios del PRP: crecimiento y maduración ósea, estabilización de injertos, cicatrización, sellado de heridas (colgajos), hemostasia. (12) 


\section{Tratamiento de la Gonartrosis mediante la aplicación de Plasma Rico en \\ Plaquetas}

Vol. 2, núm. 2., (2018)

Md José Eduardo Cedeño Gilces; Dr. Cesar Aurelio Chalen Lainez; Md. Ana María Garavito Martínez; Md. Paola Janneth Bravo Rey

El PRP actúa a través de los factores de crecimiento contenidos en las plaquetas, es una manera simple y no costosa de obtener los factores de crecimiento y citoquinas. Al activarse las plaquetas producen una respuesta de las proteínas biológicamente activas que se unen a los receptores transmembrana de las células diana que conllevan a un reclutamiento celular, crecimiento, morfogénesis y modulación de la inflamación. También tiene un efecto antibacterial. El PRP favorece y acelera la reparación de tejidos con bajo potencial de regeneración, como es el cartílago. Por eso, se está usando ampliamente el plasma como procedimiento mínimamente invasivo en la degeneración de cartílago y osteoartrosis. (1) (8) (10)

En 2005, la revisión hecha por Filardo y colaboradores, ya planteaba que el PRP era favorable para la regeneración de los tejidos articulares, no solo reparando el cartílago, también repara los tejidos meniscales y sinoviales. (1)

En 2014, se publica una revisión, luego de analizar 91 citaciones sobre PRP, se eligieron 5 estudios (dos eran randomizados y controlados, 2 eran estudios prospectivos y otro era un análisis retrospectivo). Concluyen luego de esa revisión que la inyección intraarticular (IA) de PRP reduce el dolor, considerando que es un tratamiento eficaz en la osteoartrosis de rodilla.

Plasma Rico en Plaquetas (PRP) vs Acido Hialurónico (AH)

Trabajo con 109 pacientes (55 con AH y 54 con PRP) quienes recibieron el tratamiento y fueron evaluados a los 12 meses. Los criterios de inclusión: mayor de 18 años, 4 o más meses con dolor o inflamación de rodilla e imágenes con cambios degenerativos de articulación. Un 


\section{Tratamiento de la Gonartrosis mediante la aplicación de Plasma Rico en Plaquetas}

Vol. 2, núm. 2., (2018)

Md José Eduardo Cedeño Gilces; Dr. Cesar Aurelio Chalen Lainez; Md. Ana María Garavito Martínez; Md. Paola Janneth Bravo Rey

ciclo de 3 inyecciones semanales, administradas a ciegas. Todos fueron evaluados prospectivamente a los 2, 6 y 12 meses. Los resultados sugieren que las inyecciones de PRP ofrecen una mejoría clínica mayor a los 12 meses. Pero en pacientes de edad media y signos moderados de OA el PRP no ofrece mejores resultados que el $\mathrm{AH}$, por lo cual no se considera primera línea de tratamiento. Hay resultados más promisorios en su utilización en daño articular de bajo grado, pero todavía tiene que ser confirmado. (14)

Un estudio clínico randomizado con grupo control, publicado en 2014, investigó los efectos del PRP sobre el dolor, edema, función y calidad de vida en pacientes con gonartrosis. Se dividieron en dos grupos, uno de ellos el control, cada grupo con 31 individuos, ambos con ejercicio terapéutico. El grupo de PRP recibió dos cursos de leucocitos ricos en PRP con un intervalo de 4 semanas. Se evaluaron al inicio y a los 6 meses del tratamiento. Los resultados mostraron más mejoría en el grupo PRP $(\mathrm{P}<0,05)$. Este estudio demostró que la inyección de PRP más ejercicio terapéutico puede ser más efectivo en reducir el dolor, mejorar edema y calidad de vida que el ejercicio solo. (15)

Otro trabajo clínico randomizado sin placebo, con una población de 160 pacientes que presentaban OA rodilla. 87 recibieron PRP con 2 IA con un intervalo de 4 semanas y 73 con AH tres dosis con una semana de intervalo. Todos fueron evaluados prospectivamente antes y a los 12 meses. Los resultados: el dolor mejoro en ambos grupos, pero se obtuvieron mejores resultados en el grupo de PRP. Concluyen que el PRP reduce síntomas, mejora calidad de vida y es una opción terapéutica en pacientes que no responden al tratamiento convencional. (16) 


\section{Tratamiento de la Gonartrosis mediante la aplicación de Plasma Rico en}

Plaquetas

Vol. 2, núm. 2., (2018)

Md José Eduardo Cedeño Gilces; Dr. Cesar Aurelio Chalen Lainez; Md. Ana María Garavito Martínez; Md. Paola Janneth Bravo Rey

Un estudio comparó a corto plazo los resultados de PRC y AH IA en OA temprana de rodilla. 118 pacientes, edad media 59,3 +/- 8,55 con diagnóstico clínico y radiológico de gonartrosis entre mayo y diciembre del 2013. Los pacientes recibieron PRP y AH IA por un total de tres veces con una semana de separación. 61 pacientes (102 rodillas) recibieron PRP y 57 pacientes (97 rodillas) recibieron AH. Se evaluaron pre y pos tratamiento a los 3 y 6 meses. Cuando se compararon los grupos pre tratamiento no hubo diferencias significativas; pero, a los 3 y 6 meses la diferencia entre ambos grupos si fue importante, siendo el PRP más eficiente que el AH. (8)

Un estudio en pacientes con gonartrosis severa combinó PRP intraarticular y en infiltración ósea en ambos tejidos simultáneamente. Hubo un significativo descenso del dolor con mejoría de la función articular, desde el inicio hasta la semana 24 luego del tratamiento. A la semana del tratamiento hubo descenso de las células mesenquimales de 7,98 +/-8,21 MSC/ml a $4.04 \pm$ 5.36 MSC/ $\mu \mathrm{L}$ y las formadoras de colonia disminuyeron de $601.75 \pm 312.30$ a $139.19 \pm 123.61$. Estos cambios evidencia el efecto positivo de la combinación. (17)

PRP vs AH vs Corticoesteroides (CE)

Hay 5 productos aprobados por la FDA (Food and Drug Administration) para inyección intraarticular: acetato de metilprednisolona, acetato triamcinolona, acetato de betametasona, fosfato sódico de betametasona, hexacetonida triamcinolona y dexametasona. (18)

Un trabajo indica que en la actual literatura y la experiencia de los autores, la IA es segura y tiene efectos positivos para la satisfacción del paciente. Los CS son una opción en la sinovitis aguda y persistente en pacientes que no pueden ser operados y efectivos a corto plazo. Los 


\section{Tratamiento de la Gonartrosis mediante la aplicación de Plasma Rico en Plaquetas}

Vol. 2, núm. 2., (2018)

Md José Eduardo Cedeño Gilces; Dr. Cesar Aurelio Chalen Lainez; Md. Ana María Garavito Martínez; Md. Paola Janneth Bravo Rey

autores prefieren $\mathrm{AH}$ en los obesos, mayores de 60 años, al tener un efecto a largo plazo, indicado en quienes no pueden ser intervenidos quirúrgicamente. Mejor para los que no pueden ser operados. El PRP lo usan en pacientes menores de 60, con OA moderada e índice de masa menor de 30. Si el paciente es mayor de 60, IMC mayor de 30 o tiene OA moderada aplican PRP seguido de una sola dosis de AH 2 a 4 semanas luego del PRP. (18)

El PRP en distintos estudios, ya sea como monoterapia o combinado ha demostrado ser útil en mejorar los síntomas de la osteoartrosis de rodilla, con menor riesgo de complicaciones o efectos adversos y en regenerar la articulación lesionada.

\section{Conclusiones.}

La Gonartrosis actualmente puede ser considerada un problema de salud pública, ya que limita la capacidad funcional de los pacientes y genera costos importantes al sistema de salud pública.

Al aumentar el promedio de vida, la gonartrosis es una patología con mayor incidencia, debido al mayor desgaste articular que se experimenta en la tercera edad.

Hay distintos tratamientos, desde las modificaciones del estilo de vida, bajar de peso, ejercicio moderado o adecuado a la persona; también, está el manejo farmacológico y por último el quirúrgico. 


\section{Tratamiento de la Gonartrosis mediante la aplicación de Plasma Rico en}

Plaquetas

Vol. 2, núm. 2., (2018)

Md José Eduardo Cedeño Gilces; Dr. Cesar Aurelio Chalen Lainez; Md. Ana María Garavito Martínez; Md. Paola Janneth Bravo Rey

Con la intención de demorar la cirugía, se hace uso del tratamiento farmacológico, vía oral para mejorar los síntomas como el dolor y signos como la inflamación. A eso, se une el tratamiento intraarticular, donde se ubica el PRP para regenerar la articulación.

El PRP ha demostrado ser útil, tanto para manejo del dolor, por resguardar la y regenera la articulación lesionada, demostrando que por ser autólogo, las posibilidades de rechazo del tratamiento no existen, es mínimamente invasivo y menos costoso.

Por todo lo anteriormente explicado se puede concluir que la aplicación de PRP intraarticular es un tratamiento efectivo en la gonartrosis, ofreciendo un nuevo horizonte terapéutico para esta limitante enfermedad.

\section{Bibliografía}

1. Filardo Gea. Platelet-rich plasma: why intra-articular? A systematic review of preclinical studies and clinical evidence on PRP for joint degeneration. Knee Surgery, Sports Traumatology, Arthroscopy, 23(9). 2005;: p. 2459-2474.

2. Batlle-Gualda E. Artrosis e investigación: ¿una asignatura pendiente? Rev Esp Reumatol. 2005;: p. 88-90.

3. Rodríguez J. Osteoartrosis. In Tratado de geriatria para residentes.: Manuel Anton Jimenez; 2007.

4. García S, Segur J. Gonartrosis. Med Integral 2002. 2002;: p. 98-107.

5. Pasos F. El tratamiento del dolor en la gonartrosis. Revista Dolor Clinica y Terapia. 2008.

6. Huang G, Hua S, Yang T, Ma J, Yu W, Chen X. Platelet-rich plasma shows beneficial effects for patients with knee osteoarthritis by suppressing inflammatory factors. Exp Ther Med.. 2018;: p. 3096-3102.

7. Martínez-Rodrígueza G, Simental M, Mendía J, Cavazos H. El plasma rico en plaquetas en osteoartrosis de rodilla: una alternativa de tratamiento. Artículo de revisión. Cirugía y Cirujanos. 2015;: p. 352-358.

8. Kilincoglu V, Abdurrahman Y. Short term results comparison of intraarticular platelet-rich plasma (prp) and hyaluronic acid (ha) applications in early stage of knee osteoarthritis. Int J Clin Exp Med. 2015;: p. 18807-18812.

9. López C, González M, Galnares. L. Inyección intra-articular de plasma rico en plaquetas para 


\section{Tratamiento de la Gonartrosis mediante la aplicación de Plasma Rico en Plaquetas}

Vol. 2, núm. 2., (2018)

Md José Eduardo Cedeño Gilces; Dr. Cesar Aurelio Chalen Lainez; Md. Ana María Garavito Martínez; Md. Paola Janneth Bravo Rey

el tratamiento de la artrosis de rodilla Vitoria-Gasteiz: Servicio central de Publicaciones dle Gobierno Vasco; 2014.

10. Moreno R, Gaspar M, Jiménez J, Alonso J, Villimar A, López P. Técnicas de obtención del plasma rico en plaquetas y su empleo en terapéutica osteoinductora. Farm Hosp.. 2015; 39(3).

11. Carrasco J, Bonete D, Gomar. F. Plasma Rico en Plaquetas vs. Plasma rico en factores de crecimiento. Revista Española de Cirugía Osteoarticular. 2009;: p. 127-140.

12. González M, Arteaga-Vizcaíno M, Benito M, Benito M. Aplicación del plasma rico en plaquetas (PRP) y sus derivados en implantología dental y cirugía plástica. Invest. clín. 2012; 53.

13. Anitua E, Sánchez M, Aguirre J, Prado R, Padilla S, Orive. G. Efficacy and safety of plasma rich in growth factors intra-articular infiltrations in the treatment of knee osteoarthritis. Arthroscopy. 2014;: p. 1006-17.

14. Filardo G, Kon E, Martino AD, Matteo BD, Merli M, Cenacchi A. Platelet-rich plasma vs hyaluronic acid to treat knee degenerative pathology: study design and preliminary results of a randomized controlled trial. BMC Musculoskelet Disord.. 2012 Nov; 23(13).

15. Rayegani S, Raeissadat S, Taheri M, Babaee M, Bahrami M. Does intra articular platelet rich plasma injection improve function, pain and quality of life in patients with osteoarthritis of the knee? A randomized clinical trial. Orthop Rev (Pavia). 2014.

16. Raeissadat S, Rayegani S, Hassanabadi H. Knee Osteoarthritis Injection Choices: PlateletRich Plasma (PRP) Versus Hyaluronic Acid (A one-year randomized clinical trial). Clin Med Insights Arthritis Musculoskelet Disord. 2015;: p. 1-8.

17. Sánchez M, Delgado D, Sánchez P, Muiños-López E. Combination of Intra-Articular and Intraosseous Injections of Platelet Rich Plasma for Severe Knee Osteoarthritis: A Pilot Study. Biomed Res Int. 2016.

18. Egemen A, Hayrettin K, Akgun I. Intraarticular injections (corticosteroid, hyaluronic acid, platelet rich plasma) for the knee osteoarthritis. World J Orthop. 2014;: p. 351-361.

19. A.Cortés Fabregat AHRAMIPDOP. Eficacia del tratamiento de la gonartrosis con ácido hialurónico intraarticular. valoración funcional basada en parámetros cinéticos. Rehabilitación. volumen 35. 2001;: p. 195-201.

20. Mario Alberto Simental-Mendía JFVCyHGMR. El plasma rico en plaquetas en osteoartrosis de rodilla: una alternativa de tratamiento. Artículo de revisión. CIRUGÍA y CIRUJANOS. 2015;: p. 352-358. 